\title{
Addressing tumor blood vessels
}

\author{
Judah Folkman
}

When tumors switch to the angiogenic phenotype and recruit new blood vessels, endothelial cells in these vessels express proteins on their luminal surface that are not produced by normal quiescent vascular endothelium. One of these proteins, alpha $\beta_{3}$ integrin, when prevented from binding to its ligand, causes apoptosis of endothelial cells in proliferating microvessels ${ }^{1}$. In this issue, Pasqualini et $\mathrm{al}^{2}$ report an in vivo phage display method that permits selective targeting of the alpha $_{\mathrm{v}}$ integrins by a specific, nine-residue cyclic peptide containing an ArgGlyAsp (RGD) sequence. When injected intravenously, the nonapeptide expressed on the bacteriophage surface homes to new blood vessels of murine and human tumors in mice to levels 40-80-fold greater than those in control organs. The RGD peptide has such a high affinity for alpha integrins that it localizes in the endothelial lining of the small blood vessels of tumors and is still present 24 hours after injection, at which time about $90 \%$ of it has cleared from the circulation.

This is an exciting paper that has important fundamental and clinical implications in view of certain unique aspects of growth regulation in blood vessels. Vascular endothelial cells form a monolayer covering approximately $1000 \mathrm{~m}^{2}$ in a $70 \mathrm{~kg}$ person. These cells rarely proliferate and have a mean turnover time of hundreds of days or more ${ }^{3}$. However, during angiogenesis-and especially in a neovascularized tumor-endothelial cells can proliferate as fast as bone marrow progenitor cells (e.g., with a turnover time in the range of a few days). The objective of antiangiogenic therapy is to return this focus or "hotspot" of proliferating microvessels to its normal resting state, or even destroy it. When this is accomplished by angiogenesis inhibitors

Judah Folkman is Andrus professor of pediatric surgery and professor of cell biology, Harvard Medical School, Children's Hospital Hunnewell 103, 300 Longwood Avenue, Boston, MA 02115 (Tel: 617355 7661; Fax: 6173557662 ).

potent enough to block angiogenesis completely, large bulky tumors in mice can regress to microscopic dormant tumors 4 because tumor growth is angiogenesisdependent.

The in vivo targeting system developed by Pasqualini et al. may provide a vehicle for directing endothelial inhibitors to any focus of angiogenesis, whether it is in a tumor or in the retina. For molecules that specifically

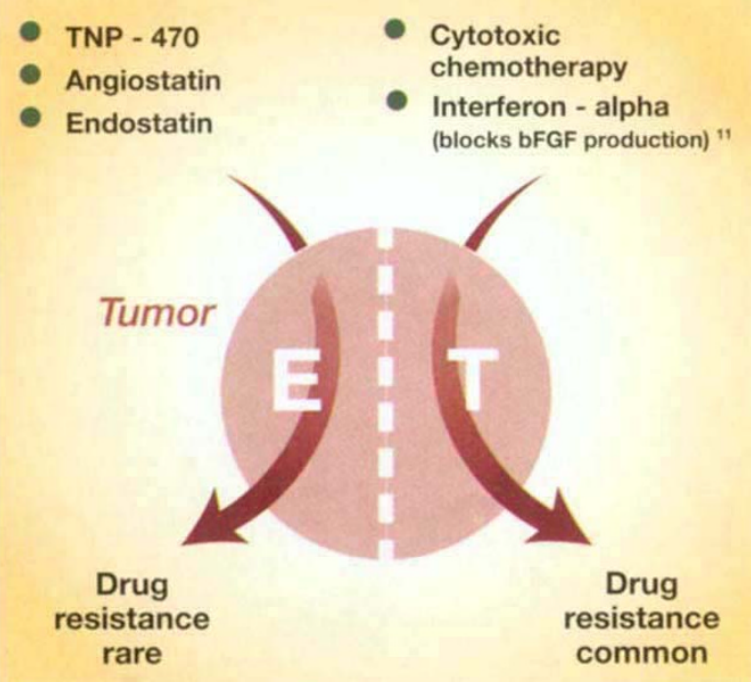

Figure 1. Is antiangiogenic therapy a strategy to bypass drug resistance? Within a tumor, the tumor cell population (T) commonly develops resistance to a wide variety of (E) does not. Thus, therapies directed against endothelial cells, rather than tumor cells, are less likely to induce drug resistance.

inhibit proliferating endothelium (e.g., angiostatin and endostatin) and antibodies) or peptides that block alpha $\beta_{3}$ integrin, a delivery method that would concentrate them in tumors could increase efficacy and decrease the amount of precious protein required for long-term therapy. Could efficacy be increased for such selective angiogenesis inhibitors as the fumagillin analog TNP-470 (ref. 5) or the bacterial-derived polysaccharide CM101 if they were complexed with the nonapeptide? Could the efficacy of antivascular therapy ${ }^{6}$ be increased by linking a tissue factor or other cytokines with the nonapeptide?

A more immediate clinical application might be a method to direct conventional cytotoxic chemotherapy to the endothelial cell population of a tumor. This strategy could lower toxicity and possibly bypass drug resistance ${ }^{7}$ (see Fig. 1). Tumor cells are genetically unstable, rapidly mutating, and able to generate drug-resistant clones, but vascular endothelial cells are genetically stable and rarely become drug resistant (in a manner analogous to bone marrow). Longterm antiangiogenic therapy directed against the endothelial cell population of a tumor has not shown drug resistance either in experimental animals or in clinical trials. Therefore, if tumor-bearing mice were treated with a conventional cytotoxic agent fused to the nonapeptide, the drug might be administered at a low dose that would avoid systemic toxicity, but allow it to concentrate in tumor vessels to control tumor growth solely by its antiendothelial effect. The drug would not need to leave the circulation and enter the tumor, nor would it have to cross the blood brain barrier.

Alternatively, it might be possible to optimize the antiangiogenic activity of the few cytotoxic drugs known to inhibit blood vessel growth. For example, if the nonapeptide were bound to paclitaxel (Taxol), which inhibits angiogenesis in animals below its cytotoxic dose ${ }^{8}$, could a Taxol-resistant tumor respond to the Taxol-nonapeptide complex?

If the nonapeptide has an affinity for new tumor vessels in a histological section, the efficacy of microvessel counts of tumor tissues as a prognostic indicator could be increased (for a recent review, see ref. 9). Studies are underway to quantify the intensity of tumor neovascularization in real time by high-resolution magnetic resonance imaging ${ }^{10}$, and these efforts may be facilitated by the targeting of alpha $\mathrm{v}_{\mathrm{v}}$ integrins with radionuclides, as the authors suggest.

Perhaps the most fundamental contribution of the report is that it provides further compelling evidence for an emerging picture of heterogeneity in vascular endothelium, heterogeneity that exists not only from one organ to another, but from proliferating to nonproliferating endothelium.

1. Brooks, P.C. et al. 1994. Cell 79:1157-1164.

2. Pasqualini, R., Koivunen, E., Ruoslahti, E. 1997. Nature Biotechnology 15:542-546.

3. Denekamp, J. 1990. Cancer Metast. Rev. 3:267-282.

4. O'Reilly, M.S. et al. 1997. Cell 88:277-285.

5.Ingter, D.E. 1997. Discovery of TNP-470 and other angiogenesis inhibitors, in Cancer Therapeutics: Experimental and Clinical Agents. Teicher, B. (ed.). Humana Press, Totowa, NJ.

6. Huang, $X$. et al. 1997. Science 275:547-550.

7. Kerbel, R.S. 1991. BioEssays 13:31-36.

8. Belotti, D. et al.. 1996. Clin. Cancer Res. 2:1843-1849.

9. Gasparini, G. 1996. Eur. J. Cancer 32A:2485-2493.

10. Esserman, L., Weidner, N., Yassa, L., Hylron, N. 1996. Proc. Am. Soc. Clin. Oncol. 15:129.

11. Singh, R.K., Gutman, M., Bucana, C.D., Sanchez, R., liansa, N., Fidler, I.J. 1995. Proc. Natl. Acad. Sci. USA 92:4562-4566. 